\title{
Tratamento fisioterapêutico com o uso da eletroestimulação funcional e a facilitação neuromuscular proprioceptiva em pacientes com sequelas de AVC
}

\author{
Physiotherapeutic treatment with the use of functional electro-stimulation and \\ proprioceptive neuromuscular facilitation in patients with stroke sequels
}
Tratamiento fisioterapéutico con el uso de electroestimulación funcional y facilitación neuromuscular proprioceptiva en pacientes con secuelas de ACV

Francisco Jhonatan Sousa da Silva ${ }^{1 *}$, Maria Isabelle das Neves Menezes ${ }^{1}$, Maria Leandra de Souza Silva ${ }^{1}$, Valéria Pereira Alves ${ }^{1}$.

\section{RESUMO}

Objetivo: O objetivo desse estudo é analisar as evidências científicas encontradas sobre o tratamento do AVC através da eletroestimulação funcional (FES) e da facilitação neuromuscular proprioceptiva (FNP). Métodos: A metodologia usada foi uma revisão de literatura integrativa, nas bases de dados, SCIELO, PEDro, artigos publicados nas bases de dados nos últimos 10 anos. Foram avaliados os efeitos da eletroestimulação funcional (FES) e da facilitação neuromuscular proprioceptiva (FNP) nos pacientes com sequelas de AVC. Resultados: A busca resultou no total de 357 artigos que após os critérios de inclusão e exclusão ficaram 10 artigos para o estudo onde foi dividido em dois quadros ressaltando os tratamentos e sua eficácia onde, ao total foram analisados dez artigos dos quais seis são estudos piloto, 1 ensaio clinico, 2 estudos controle e 1 estudo transversal. Considerações Finais: Com a aplicação dos dois recursos, foi observado que os mesmos removem uma reabilitação mais eficaz, proporcionando maior qualidade de vida aos pacientes. Sugere-se que estudos mais complexos sejam realizados, com amostras maiores e mais homogêneas, na tentativa de análises mais eficientes.

Palavras-chave: Acidente vascular cerebral, Eletroterapia, Propriocepção, Terapia por exercício, Estimulação elétrica.

\section{ABSTRACT}

Objective: The objective of this study is to analyze the scientific evidence found on the treatment of stroke through functional electrostimulation (FES) and proprioceptive neuromuscular facilitation (PNF). Methods: The methodology used was an integrative literature review, in the databases, SCIELO, PEDro, articles published in the databases in the last 10 years. The effects of functional electrostimulation (FES) and proprioceptive neuromuscular facilitation (PNF) were evaluated in patients with stroke sequelae. Results: The search resulted in a total of 357 articles that after the inclusion and exclusion criteria were 10 articles for the study where it was divided into two frames highlighting the treatments and their effectiveness where, in total, ten articles were analyzed, of which six are pilot studies, 1 clinical trial, 2 control studies and 1 cross-sectional study. Final Considerations: With the application of both resources, it was observed that they remove a more effective rehabilitation, providing greater quality of life to patients. It is suggested that more complex studies be performed, with larger and more homogeneous samples, in an attempt to more efficient analyzes.

Keywords: Stroke, Electrotherapy, Proprioception, Exercise therapy, Electrical stimulation.

\section{RESUMEN}

Objetivo: El objetivo de este estudio es analizar la evidencia científica encontrada sobre el tratamiento del ictus mediante electroestimulación funcional (FES) y facilitación neuromuscular propioceptiva (FNP). Métodos: La metodología utilizada fue una revisión integradora de la literatura, en las bases de datos, SCIELO, PEDro, artículos publicados en las bases de datos en los últimos 10 años. Se evaluaron los efectos de la electroestimulación funcional (FES) y la facilitación neuromuscular propioceptiva (PNF) en pacientes con secuelas de ictus.

\footnotetext{
${ }^{1}$ Faculdade de Medicina Estácio Juazeiro do Norte (FMJ), Juazeiro do Norte - CE.
}

*E-mail: johnathansousa6@gmail.com 
Resultados: La búsque da resultó en un total de 357 artículos que luego de los criterios de inclusión y exclusión quedaron 10 artículos para el estudio donde se dividió en dos quadros destacando los tratamientos y su efectividad donde, en total, se analizaron diez artículos, de los cuales seis son estudios piloto, 1 ensayo clínico, 2 estudios de control y 1 estudio transversal. Consideraciones Finales: Con la aplicación de ambos recursos se observó que eliminan una rehabilitación más efectiva, brindando mayor calidad de vida a los pacientes. Se sugiere que se realicen estudios más complejos, con muestras más grandes y homogéneas, en un intento de análisis más eficientes.

Palabras clave: Ictus, Electroterapia, Propiocepción, Terapia de ejercicio, Estimulación eléctrica.

\section{INTRODUÇÃO}

O Acidente Vascular Cerebral (AVC) é uma doença neurológica que acontece quando o suprimento de sangue que chega ao cérebro é interrompido levando a uma isquemia (AVC Isquêmico) ou quando um vaso sanguíneo se rompe nessa região, causando uma hemorragia cerebral (AVC Hemorrágico) (BRASIL, 2017). No AVC há comprometimentos neurológicos e déficits sensoriomotores que causam alterações como hemiplegia ou hemiparesia, distúrbios da coordenação e sensibilidade que podem gerar limitação das atividades de vida diárias (AVD), e incapacidades funcionais limitando a vida social dos pacientes (MARTIN ST e KESSLER M, 2017).

De acordo com Barbosa DJ, et al. (2015), em média uma em cada seis pessoas no mundo terá um AVC. Porém, em muitos casos acontece apenas um AIT (Ataque Isquêmico Transitório) que é definido como uma rápida perda da função cerebral e dura geralmente entre 10 a 20 minutos com recuperação completa dos sintomas. O AVC é a segunda principal causa de mortes no mundo e, em alguns casos, deixa o paciente com incapacidade de realizar suas atividades diárias. Aproximadamente $85 \%$ dos casos de Acidente vascular cerebral são isquêmicos e apenas 15\% hemorrágicos (BRASIL, 2017).

A Facilitação Neuromuscular Proprioceptiva (FNP) é um método fisioterapêutico que utiliza movimentos funcionais em diagonais que tem por objetivo a facilitação dos movimentos, fortalecimento muscular e ganho no controle da coordenação dos movimentos (MAGDALON EC, 2014). Outro recurso é a Eletroestimulação Funcional (FES) que atua recuperando a funcionalidade do paciente, estimulando fibras musculares a produzir contrações através de uma corrente elétrica, promovendo assim um aumento da força muscular e contribuindo na normalização da espasticidade (MILESKI ME, et al., 2013).

Esse trabalho será de grande importância para a melhora da qualidade de vida dos pacientes acometidos pós-lesão do AVC, com a possibilidade dos profissionais da fisioterapia ampliarem o seu conhecimento acerca das possibilidades de tratamento através a eletroestimulação funcional (FES) e da facilitação neuromuscular proprioceptiva (FNP) propondo uma reabilitação eficaz com alta adesão, baixo custo e maior recuperação da função, promovendo recuperação física e mental. Diante do exposto acima, o objetivo dessa pesquisa é analisar nas principais bases de dados as publicações de maior relevância sobre o tratamento da eletroestimulação funcional FES e a facilitação neuromuscular proprioceptiva FNP em pacientes com sequelas de AVC.

\section{MÉTODOS}

O presente estudo classifica-se Como exploratório, do tipo revisão de literatura integrativa, de natureza bibliográfica. O estudo exploratório é definido como a parte integrante da pesquisa principal, como um estudo preliminar que é realizado com a finalidade de melhor apropriar o que se pretende conhecer (PIOVESAN A e TEMPORINI ER, 1995).

Para Souza MT, et al. (2010) e Gil AC (2010) a revisão integrativa trata-se de um estudo de coleta de dados que é realizado a partir de fontes secundárias, baseado em estudos bibliográficos. Já a pesquisa bibliográfica é o tipo de pesquisa elaborada em bases de matérias já publicadas, nessas pesquisas inclui-se material impresso, livros, revistas, jornais, teses e dissertações. 


\section{Período de realização do estudo}

O período de coletas de informações foi realizado a partir de conteúdos já existentes como artigos científicos. Iniciou-se em fevereiro de 2019 se estendendo até novembro de 2019, sendo o período de agosto a outubro realizado a coleta de dados.

\section{Critérios de elegibilidade dos artigos}

Para a realização desse estudo foram obtidas buscas nas seguintes bases de dados: A Scientific Electronic Library Online (SciELO), Physiotherapy Evidence Database (PEDro), sendo usada nesse processo de pesquisas os seguintes descritores: Acidente Vascular Cerebral/Stroke, Eletroterapia/Electrotherapy, Propriocepção/Proprioception, Terapia por exercício/Exercise therapy, Estimulação elétrica/Electrical stimulation.

\section{Critérios de inclusão}

Os critérios de inclusão selecionados nessa pesquisa foram os artigos científicos circulares nacionais e internacionais, artigos de intervenção relacionados com o tema abordado, artigos publicados nas bases de dados supracitados nos últimos 10 anos.

\section{Critérios de exclusão}

Foram excluídos os artigos de revisão, artigos pagos, artigos que não apresentem pelo menos dois dos descritores supracitados, artigos fora do intervalo dos anos citado.

\section{Coleta de dados}

Para o desenvolvimento desta pesquisa foram elaboradas etapas que serão a base do seu processo de construção. Ela terá inicio na definição do tema seguido pelas buscas por artigos sobre AVC, Eletroterapia e Propriocepção, de acordo os critérios supracitados, mediante essa procura serão selecionados apenas os artigos que apresentem conteúdo relacionado ao tema. Após essa etapa foi feita uma leitura aprofundada dos artigos, onde foram selecionados e retirados os dados principais para as informações a ser estudada, enfatizando sobre a eletroterapia e terapia por exercício como recursos fisioterapêuticos no seu tratamento do AVC. Foi elaborado pelo pesquisador um fluxograma explicativo sobre a coleta de dados (Figura 1).

Figura1 - Fluxograma representativo das fases da coleta de dados

\section{Fase 1}

-Procura pelos artigos sobre AVC, Estimulação elétrica, FNP.

Fase 2

-Distinção dos artigos relacionados com os descritores acima citados.

\section{Fase 3}

-Seleção dos artigos de intervenção do FES de intervenção do FNP.

\section{Fase 4}

- Leitura aprofundada sobre os artigos selecionados.

\section{Fase 5}

- Retirada dos dados enfatizando no resultados e tratamento.

Fonte: Silva FJS, et al., 2020. 


\section{Análise dos dados}

$\mathrm{Na}$ etapa subsequente, após uma leitura profunda e detalhada dos artigos pertinentes a esta pesquisa, foi elaborada um quadro no Microsoft Word $2010 \mathrm{com}$ a finalidade de apresentar os dados mais significativos e classificativos de cada um deles. O quadro apresentou os artigos mediantes os seguintes conteúdos: autor/ano, objetivo do estudo, tipo de estudo, população ou amostra estudada e principais resultados.

\section{RESULTADOS E DISCUSSÃO}

As buscas resultaram em um total de 357 artigos (49 SCIELO e PEDro 308) onde após aplicados os critérios de inclusão e exclusão, restaram apenas 10 artigos, dos quais foram 4 SCIELO e 6 PEDro. O fluxograma a seguir demonstra o processo de seleção e exclusão e inclusão dos artigos.

Figura 2 - Fluxograma demonstrativo sobre o processo de seleção, exclusão e inclusão dos artigos.

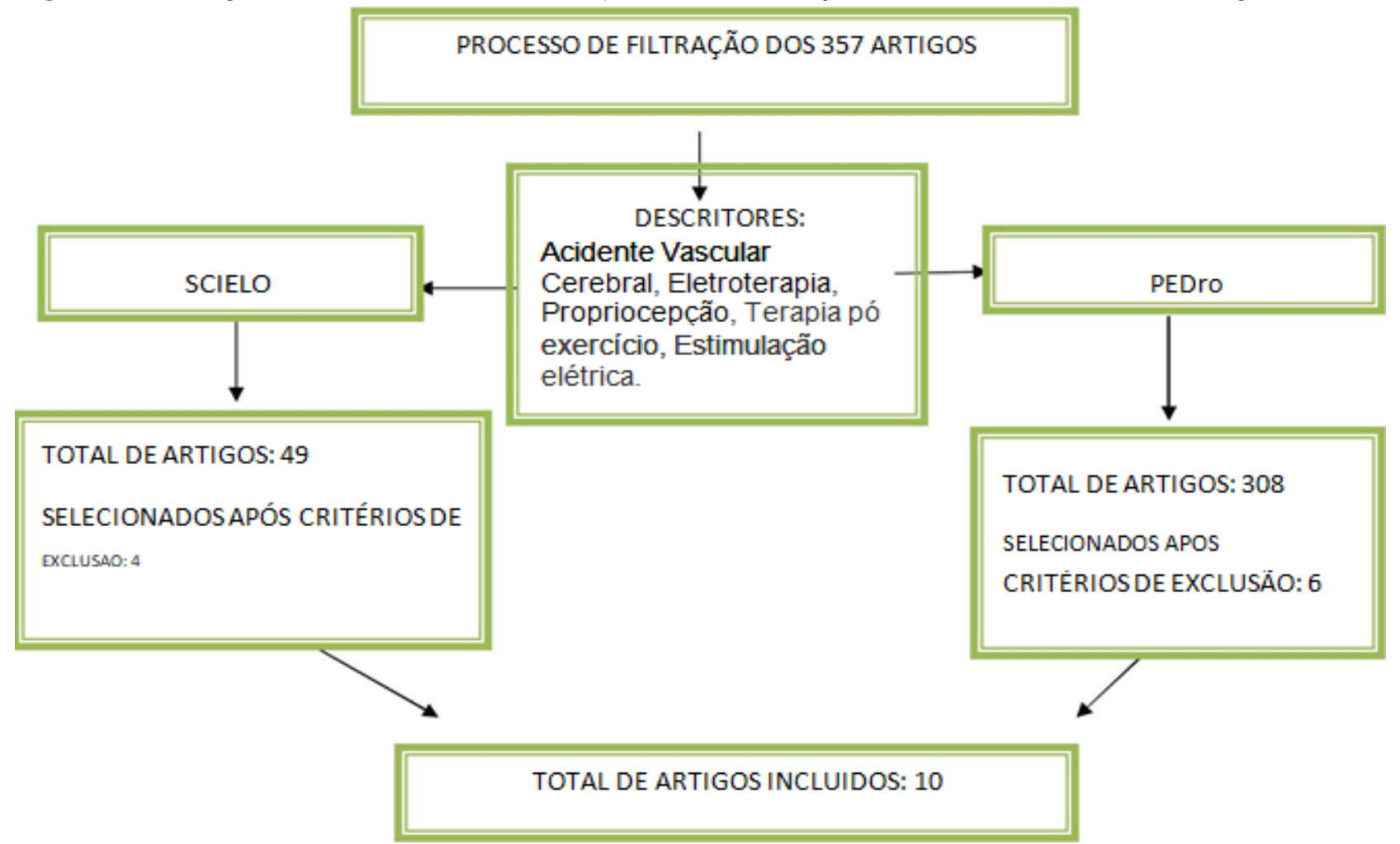

Fonte: Silva FJS, et al., 2020.

O Quadro 1 mostrará de forma resumida os dados mais significativos dos artigos selecionados para este estudo de revisão integrativa com os seguintes conteúdos: título do artigo, autor/ano, tipo de estudo, objetivo e principais resultados. 


\section{Revista Eletrônica Acervo Saúde / Electronic Journal Collection Health | ISSN 2178-2091}

Quadro 1 - Intervenção da Eletroestimulação elétrica (FES) nas sequelas do AVC

\begin{tabular}{|c|c|c|c|c|}
\hline TITULO DO ARTIGO & AUTOR $\backslash$ ANO & TIPO DE ESTUDO & OBJETIVO & PRINCIPAIS RESULTAOS \\
\hline $\begin{array}{l}\text { Treinamento motor da } \\
\text { extremidade superior com } \\
\text { estimulação elétrica funcional } \\
\text { na reabilitação precoce no } \\
\text { AVC. }\end{array}$ & $\begin{array}{l}\text { (MAGDALON EC, et } \\
\text { al., 2009). }\end{array}$ & Estudo piloto & $\begin{array}{l}\text { Investigar o efeito do tratamento } \\
\text { motor com FES na recuperação } \\
\text { motora do braço e mão em pacientes } \\
\text { com AVC agudo, subaguda e grave. }\end{array}$ & $\begin{array}{l}\text { O estudo foi dividido em dois grupos o } \\
\text { controle e o FES, os resultados foram mais } \\
\text { satisfatórios no grupo FES no Indice de } \\
\text { Barthel - EBI de braço e mão obteve } \\
\text { melhores resultados significativamente. }\end{array}$ \\
\hline $\begin{array}{l}\text { Estimulação elétrica } \\
\text { funcional na recuperação da } \\
\text { extremidade superior durante } \\
\text { a reabilitação do AVC. }\end{array}$ & $\begin{array}{c}\text { (ALON G, et al., } \\
\text { 2007). }\end{array}$ & Estudo piloto & $\begin{array}{l}\text { Testar se a estimulação elétrica funci- } \\
\text { onal (FES) pode aumentar a recupe- } \\
\text { ração da função da extremidade } \\
\text { superior durante a reabilitação } \\
\text { precoce. }\end{array}$ & $\begin{array}{l}\text { A pontuação média do teste Box \& Blocos- } \\
\text { B\&B em } 12 \text { semanas favoreceu o grupo FES } \\
\text { com } 16,6 \text { blocos e o grupo controle apenas } \\
\text { com } 11,0 \text {, porem os dos grupos obtiveram } \\
\text { respostas na recuperação da função da } \\
\text { mão. }\end{array}$ \\
\hline $\begin{array}{l}\text { Treinamento bilateral dos } \\
\text { membros superiores com } \\
\text { estimulação elétrica funcional } \\
\text { em pacientes com AVC } \\
\text { crônico. }\end{array}$ & $\begin{array}{l}\text { (LEUNG C, et al., } \\
\text { 2009). }\end{array}$ & Estudo controlado & $\begin{array}{l}\text { Investigar a eficácia da estimulação } \\
\text { elétrica funcional (FES) com } \\
\text { atividades bilaterais visando a função } \\
\text { do membro superior. }\end{array}$ & $\begin{array}{l}\text { O grupo FES após } 15 \text { sessões foi quem } \\
\text { obteve melhores resultados em } \\
\text { movimentação do punho quando } \\
\text { comparado ao grupo controle. }\end{array}$ \\
\hline $\begin{array}{l}\text { Efeitos da estimulação } \\
\text { elétrica funcional em função } \\
\text { do punho espastico no AVC. }\end{array}$ & $\begin{array}{l}\text { (KARAKUŞ D, et al., } \\
\text { 2013). }\end{array}$ & $\begin{array}{l}\text { Estudo rando- } \\
\text { mizado controlado }\end{array}$ & $\begin{array}{l}\text { Investigar o efeito da estimulação } \\
\text { elétrica funcional em função do punho } \\
\text { espastico, em indivíduos com AVC } \\
\text { subagudo e crônico. }\end{array}$ & $\begin{array}{l}\text { Entre os dois grupos no presente estudo não } \\
\text { houve resultados estatisticamente signifi- } \\
\text { cantes, porem o grupo da eletroestimulação } \\
\text { obteve melhores resultados. }\end{array}$ \\
\hline
\end{tabular}

Fonte: Silva FJS, et al., 2020. 
Em sua pesquisa Magdalon EC, et al. (2009), analisou vinte e três participantes divididos em dois grupos, 11 no grupo controle onde foi avaliada nesses pacientes a mão dominante com sequela pós AVC e 12 no grupo do FES que por fim foi avaliado a mão não dominante desses indivíduos.

Estes foram avaliado pela pontuação do Índice Estendida de Barthel (EBI) onde foi analisado o desempenho das atividades da vida diária (AVD). Todos os pacientes completaram o estudo, os do grupo FES obtiveram um total de 10 a 20 sessões, os participantes e grupo controle tinham 8 a 15 sessões de terapia.

De acordo com autor supracitado o grupo FES mostrou melhoras em todas as medidas de resultados primários, e seu Índice Estendida de Barthel- EBI no subescore de braço e mão teve maior relevância, pois demonstrou um maior desenvolvimento quanto ao grupo controle, que não tive resultados com nível de significância no EBI, assim não obtendo resultados na função da mão (MAGDALON EC, et al., 2009).

Segundo uma pesquisa similar Ratnasabapathy $Y$, et al. (2003), mostra que a pontuação nos níveis de subescore de mão no grupo controle apresentou o Índice Estendida de Barthel- EBI significativamente menor do que o grupo FES.

O EBI é uma avaliação bimanual, então o subescore EBI no grupo controle que avaliou a mão dominante teve menores valores por ser mais afetadas, este pode ser o motivo a qual o grupo controle tivesse com menor destreza do que o grupo FES que avaliou mão não dominante resultando assim em melhores resultados ocasionando um potencial de melhoria mais significante.

Segundo Alon G, et al. (2007), participaram desse estudo quarenta e um pacientes com sequela de AVC isquêmico, todos estando no primeiro quadro de AVC. Vinte e seis $(63,4 \%)$ pacientes foram excluídos por causa de distúrbios motores graves com perda da função da extremidade superior e menor probabilidade de recuperação da função da mão, restando apenas 15 indivíduos que foram divididos em grupo controle e grupo FES.

Ainda no presente estudo de Alon G, et al. (2007), o grupo controle seguiu um procedimento padronizado e específico de treinamento físico (PT) e ocupacional (TO), já o grupo FES seguiu as mesmas instruções PT / TO padronizada e específica que foi sincronizada com a estimulação elétrica e contração induzida padronizada para contração dos flexores e extensores do punho / dedo para auxiliar a abrir e fechar a mão parética.

Houve limitações quanto ao número de sessões compostas entre os dois grupos, pois, os pacientes estavam no período de pós-AVC agudo e subagudo e tinham suas limitações quanto a execução dos exercícios por um longo período de tempo.

Foram realizados dois testes: o de Box \& Blocks-B\&B, onde foi realizado com uma caixa contendo 150 blocos localizados em uma mesa de frente para o paciente, estes foram instruídos a pegar um bloco de cada vez e transferi-lo para o outro lado da caixa o mais rápido possível e repetindo a atividade por 60 segundos. $O$ teste $\mathrm{f}$ oi repetido 3 vezes com cada mão e as pontuações mais altas alcançadas foram o resultado final para cada mão (ALON G, et al., 2007).

Um segundo teste foi o de elevação de objetos leves Jebsen-Taylor (J-T), este avalia a capacidade de agarrar, segurar, mover e colocar grandes objetos. Cada participante sentou- se diante de 5 latas de alumínio vazias colocados em uma fileira de $5 \mathrm{~cm}$ de distância na frente de uma placa que quantificou a recuperação da função da extremidade superior.

Os pacientes foram instruídos a mover as latas o teste foi repetido 3 vezes em cada mão, o tempo mais rápido registrado para cada mão foi o desfeche final. O teste de elevação de objetos leves $\mathrm{J}$-T foi a mais difícil tarefa funcional a ser executada, pois exigia a capacidade de extensão quase total de todos os 5 dedos para agarrar e levantar as latas do teste.

No início do teste, o tempo de realização do teste J-T foi melhor no grupo FES do que no grupo controle, ao final houve diminuição em ambos, porém, o grupo FES permaneceu tendo os melhores resultados. (ALON G, et al., 2007). 
A recuperação da função da extremidade no grupo controle pode ser justificada por Shepherd RB (2001), este demonstrou que alguns pacientes com primeiro quadro de AVC têm maior probabilidade de recuperar a extremidade superior e sua função, porém, nesta pesquisa o grupo FES demonstra uma melhor recuperação funcional ocasionado em resultados mais satisfatórios.

No estudo de Chan ML, et al. (2009), participaram vinte pacientes acometidos pelo AVC, todos eles completaram 15 sessões de treinamento com avaliações antes e após o tratamento. Foram divididos sem dois grupos: o controle e o FES, onde obtiveram 10 participantes em cada.

Foi incluído o Teste Funcional para Hemiplégica de Extremidade superior (FTHUE), avaliação de FuglMeyer (FMA), força de preensão, teste de alcance amplitude de movimento ativa do punho em extensão, medida de independência funcional e Escala de Ashworth modificada.

Ainda relacionado com o estudo de Chan ML, et al. (2009), o grupo FES após a intervenção foi o que demonstrou melhora no controle de membro superior, amplitude de movimento ativa em extensão do punho e força, embora o grupo controle tenha obtido alguns resultados positivos pós-tratamento.

Ao comparar o pré e pós- teste entre os dois grupos, não houve diferença estatística, contundo, o grupo FES mostrou uma pontuação de pós-tratamento mais elevado na função motora do membro superior hemiplégico, e uma maior amplitude de movimento ativo de extensão do punho do que o grupo controle.

O desenvolvimento do grupo FES na amplitude de movimento mostrou uma melhora significativa e os seus resultados, podem ser justificados pela estimulação elétrica em grupos musculares específicos que poderia facilitar o uso do lado afetado nas tarefas dos membros superiores.

Segundo Taub E, et al. (1993), a utilização da eletroestimulação não poderia inibir o tônus espastico da mão afetada, mas pode melhorar a movimentação para que os pacientes usem o lado afetado na realização de tarefas fazendo assim com que retornem as suas atividades diárias sem limitação.

No estudo de Karakus D, et al. (2013), foram avaliados vinte e oito pacientes acometidos pelo AVC, estes foram divididos em programa de reabilitação padrão e programa de reabilitação padrão + grupos de estimulação elétrica funcionais, ou seja, um grupo controle e um grupo do FES estes foram avaliados pela escala de Ashworth no início e duas semanas após o tratamento.

Embora não houvesse diferenças estatísticas entre os grupos, houve uma melhoria que estava próxima da significância estatística no tratamento de programa funcional padrão + grupo da estimulação elétrica funcional.

Segundo Brashear A, et al. (2002), a escala de Ashworth é desenvolvida para avaliar a espasticidade, e é uma escala fácil de usar, válida e amplamente utilizada para avaliar o resultado do tratamento, contudo ela apresenta baixa sensibilidade a níveis científicos já que demonstra grande variabilidade subjetiva como á velocidade e força que o avaliador aplica o movimento e a experiência do aplicador. Isso justifica a ausência de diferença estatística (KARAKUS D, et al., 2013).

No índice de motricity ambos os grupos também não tiveram nenhuma diferença estatisticamente significativas após o tratamento, de acordo com Bohannon RW (1999). O uso da eletroestimulação funcional FES gera uma contração involuntária da musculatura levando a um ganho no fortalecimento muscular e minimizando a espasticidade.

Os artigos supracitados mostram que o grupo FES obteve resultados em ganho de movimentação em braço e mão, recuperação da função da extremidade, melhora no controle do membro superior, amplitude de movimento em extensão do punho e força quando comparados aos resultados no grupo controle. 


\section{Revista Eletrônica Acervo Saúde / Electronic Journal Collection Health | ISSN 2178-2091}

Quadro 2 - Intervenção da Facilitação Neuromuscular Proprioceptiva FNP em pacientes com sequelas de AVC.

\begin{tabular}{|c|c|c|c|c|}
\hline Titulo do artigo & Autorlano & Tipo de artigo & Objetivo & Principais resultados \\
\hline $\begin{array}{l}\text { Facilitação Neuromuscular } \\
\text { Proprioceptiva na marcha em } \\
\text { pacientes com sequelas de } \\
\text { acidente vascular encefálico. }\end{array}$ & $\begin{array}{c}\text { (SANTOS NS, et al., } \\
\text { 2016). }\end{array}$ & $\begin{array}{c}\text { Estudo } \\
\text { Transversal }\end{array}$ & $\begin{array}{l}\text { Avaliar os efeitos da Facilitação neuro- } \\
\text { muscular proprioceptiva na marcha em } \\
\text { pacientes com Acidente vascular } \\
\text { encefálico. }\end{array}$ & $\begin{array}{l}\text { Os pacientes do presente estudo obtiveram } \\
\text { melhoras na amplitude de movimento no mem- } \\
\text { bro hemiparético e melhoras no movimento de } \\
\text { dorsoflexão. }\end{array}$ \\
\hline $\begin{array}{l}\text { Os efeitos do exercício de } \\
\text { estabilidade de tronco usando } \\
\text { FNP no teste de alcance } \\
\text { funcional e nas atividades } \\
\text { musculares de pacientes com } \\
\text { AVC. }\end{array}$ & (KIM Y, et al., 2011). & Ensaio clínico & $\begin{array}{l}\text { Investigar os efeitos do exercício de } \\
\text { estabilidade do tronco, utilizando a } \\
\text { técnica de facilitação neuromuscular } \\
\text { proprioceptiva FNP sobre a ativação } \\
\text { muscular em pacientes com AVC e os } \\
\text { seus resultados no teste de alcance } \\
\text { funcional (FRT). }\end{array}$ & $\begin{array}{l}\text { Os resultados desse estudo demonstram que, } \\
\text { após a realização do programa de exercícios } \\
\text { terapêuticos, o grupo experimental mostrou } \\
\text { melhoras significantes. }\end{array}$ \\
\hline $\begin{array}{l}\text { Efeitos da facilitação neuro- } \\
\text { muscular proprioceptiva na } \\
\text { estabilidade postural e risco de } \\
\text { quebras em pacientes com } \\
\text { AVC. }\end{array}$ & $\begin{array}{l}\text { (LARCEDA NN, et } \\
\text { al., 2013). }\end{array}$ & Estudo piloto & $\begin{array}{l}\text { Avaliar o efeito do método facilitação } \\
\text { neuromuscular proprioceptiva FNP na es- } \\
\text { tabilidade postural e risco de quedas em } \\
\text { pacientes com sequelas de AVC. }\end{array}$ & $\begin{array}{l}\text { Foram atendidos } 12 \text { homens com hemiparesia } \\
\text { à esquerda e observou-se diferença altamente } \\
\text { significativa entre os valores pré e pos testes } \\
\text { por meio da Escala de Equilíbrio de } \\
\text { BERG - EEB. }\end{array}$ \\
\hline $\begin{array}{l}\text { Os efeitos do exercício de } \\
\text { marcha em rampa com FNP no } \\
\text { equilíbrio dinâmico dos } \\
\text { pacientes com AVC. }\end{array}$ & $\begin{array}{c}\text { (CHUL S e KIM A } \\
\text { 2015). }\end{array}$ & Ensaio clínico & $\begin{array}{l}\text { Examinar os efeitos dos exercícios de } \\
\text { marcha em rampa utilizando padrões de } \\
\text { facilitação neuromuscular proprioceptiva } \\
\text { FNP nas extremidades no equilíbrio } \\
\text { dinâmico e capacidade dos pacientes } \\
\text { com AVC crônicos. }\end{array}$ & $\begin{array}{l}\text { Após a investigação, haviam aumentado } \\
\text { significativamente no grupo experimental, no } \\
\text { entanto, os valores não mostraram diferenças } \\
\text { significativas no gripo controle, as diferenças } \\
\text { entre os dois grupos antes das investigação e } \\
\text { após a investigação não foram significativas. }\end{array}$ \\
\hline $\begin{array}{l}\text { Efeito do fortalecimento do } \\
\text { núcleo com proprioceptivo } \\
\text { pélvico e facilitação } \\
\text { neuromuscular } \\
\text { proprioceptiva pélvico e tronco, } \\
\text { equilíbrio, marcha e função no } \\
\text { AVC crônico. } \\
\end{array}$ & $\begin{array}{c}\text { (SHARMA V e KAUR } \\
\text { J 2017). }\end{array}$ & Ensaio clínico & $\begin{array}{l}\text { Avaliar os efeitos do fortalecimento do } \\
\text { tronco combinado com a facilitação } \\
\text { neuromuscular proprioceptiva FNP sobre } \\
\text { o comprometimento do tronco, equilíbrio, } \\
\text { marcha e capacidade funcional de } \\
\text { pacientes com AVC crônico. }\end{array}$ & $\begin{array}{l}\text { A estabilização do tronco combinada com FNP } \\
\text { pélvico foi mais eficaz para melhorar o } \\
\text { comprometimento do tronco, equilíbrio e da } \\
\text { marcha de pacientes com AVC crônico. }\end{array}$ \\
\hline $\begin{array}{l}\text { Os efeitos do treinamento da } \\
\text { marcha usando facilitação } \\
\text { neuromuscular proprioceptiva } \\
\text { na capacidade de equilíbrio } \\
\text { dinâmico dos pacientes com } \\
\text { AVC. }\end{array}$ & $\begin{array}{l}\text { (CHUI S, et al., } \\
\text { 2015). }\end{array}$ & Ensaio clínico & $\begin{array}{l}\text { Analisar as mudanças dos pacientes com } \\
\text { AVC na capacidade de equilíbrio } \\
\text { dinâmico através do treino de marcha em } \\
\text { escada associado à facilitação } \\
\text { neuromuscular proprioceptiva FNP. }\end{array}$ & $\begin{array}{l}\text { O desempenho do equilíbrio dos pacientes } \\
\text { com AVC através do treinamento da marcha } \\
\text { em escadas, com o Berg Escala de Equilíbrio- } \\
\text { BBS e os resultados do teste de alcance } \\
\text { funcional- FRT aumentaram significativamente } \\
\text { e o resultado do teste de TUG diminuiu } \\
\text { significativamente no grupo experimental. }\end{array}$ \\
\hline
\end{tabular}

Fonte: Silva FJS, et al., 2020. 
Em sua pesquisa Santos NS, et al. (2016), avaliaram cinco participantes com sequelas motoras após quadro de AVC, sendo 4 mulheres e 1 homem com idade de 49 a 69 anos, apresentado tempo médio de 22,2 meses de lesão. Na entrevista as queixas mais recorrentes eram fadiga ao locomover-se ou realizar alguma tarefa rotineira, sensação de peso ao deambular e dificuldade na elevação do pé do chão. Na avaliação física todos apresentaram redução de amplitude de movimento (ADM) na flexão de quadril e joelho e na dorsiflexão do lado hemiparético, além da marcha ceifante.

O protocolo elaborado foi de 5 sessões por semanas, num total de 10 atendimentos, com a aplicação da técnica FNP baseada nas diagonais específicas para gerar ativação dos músculos extensores da articulação do quadril. Pesquisadores como Sheard PW e Paine TJ (2010), descobriram que há uma melhora de aproximadamente $60 \%$ da contração isométrica voluntaria e aumento da amplitude de movimento do quadril após a intervenção com essa técnica.

No presente estudo Santos NS, et al. (2016), os pacientes apresentaram grande evolução na marcha, isso foi quantificado através da escala de equilíbrio de BERG e o Índice de Marcha Dinâmica, onde houve mudança na contração isométrica voluntaria e aumento na amplitude de movimento ocasionando na melhora da marcha, além disso, houve também melhora na conscientização e funcionalidade da dorsiflexão.

Segundo os autores Kim Y, et al. (2011), em seu estudo realizado com quarenta participantes com diagnóstico clínico de hemiplegia pós-lesão do AVC, sendo estes divididos em grupo controle e FNP, cada um com vinte pacientes. O grupo experimental (FNP) demonstrou aumentos significativos, enquanto o grupo controle não teve qualquer diferença sustentando os resultados da pesquisa (SANTOS NS, et al., 2016).

Ainda sobre esses autores Kim Y, et al. (2011), houve a comparação da atividade do músculo sóleo durante o teste de alcance funcional (FRT) antes e depois da intervenção nos dois grupos. Com relação ao lado hemiplégico houve aumento da ativação no grupo experimental (FNP) e o grupo controle não apresentou mudanças significativas, já o lado não-hemiplégico houve redução da atividade do músculo Sóleo tanto no grupo experimental como no grupo controle. Entretanto não houve diferenças estatísticas entre os grupos avaliados.

Com relação ao lado não hemiplégico, a redução da ativação do músculo Sóleo nesses grupos pode estar relacionada ao excesso de atividade que esse lado realiza após o AVC, por conta da sobrecarga dos músculos do lado oposto. Segundo os autores Garland SG, et al. (1997), o lado afetado da extremidade inferior mostrou alta atividade de apenas o isquiotibiais e o lado não afetado da extremidade inferior apresentou baixa atividade do sóleo e moderadamente elevada atividade do músculo tibial anterior.

No estudo de Larceda NN, et al. (2013), doze homens que apresentavam hemiparesia esquerda foram submetidos a uma intervenção que consistiu em treinamento da estabilidade postural por meio de um protocolo fixo de 5 exercícios utilizando o método FNP, foram realizados 10 atendimentos, três vezes por semana e duração em média de 45 minutos.

De acordo com Larceda NN, et al. (2013), utilizou-se para avaliação a escala de equilíbrio de Berg (EEB), o Teste de Alcance Funcional e o Time Up And Go (TUG). A primeira é uma escala utilizada para avaliar o equilíbrio de pacientes neurológicos, e a segunda avalia o controle postural no alcance e a última é um método eficaz para avaliar movimentos funcionais, equilíbrio dinâmico e capacidade de marcha em relação ao tempo de execução. Após a intervenção, os valores do teste de alcance funcional e da Escala de Equilíbrio de BERG no grupo experimental tinham aumentado significativamente e o valor do tempo de execução no TUG havia diminuído, contrastando com o grupo de controle que não mostra diferença significativa. Concluíram que houve diferenças estatísticas no uso do protocolo de FNP nos indivíduos com hemiparesia em relação ao equilíbrio, controle postural, velocidade da marcha e estabilidade do tronco.

Os autores Kim Y, et al. (2011), também observaram efeitos positivos na estabilidade do tronco e na ativação muscular, mensurada por meio de eletromiografia, com utilização de protocolo de FNP em comparação ao grupo controle que realizava exercícios com caneleira. Assim como Kyo CSC e Kim H (2015), onde o grupo experimental recebeu tratamento por exercício + treinamento de marcha em rampa com FNP, e o grupo controle realizou exercício + marcha no solo, todos por 30 minutos, obtendo resultados semelhantes aos autores supracitados evidenciando a superioridade do grupo FNP. 
Isso levanta questionamentos importantes sobre o papel da facilitação Neuromuscular Proprioceptiva em extremidades como tratamento para o controle postural, já que a técnica promove uma organização neuromuscular a nível proximal possivelmente promovendo uma ativação de musculatura estabilizadora do tronco e das cinturas escapular e pélvica, auxiliando na capacidade desse tronco em reagir aos desequilíbrios.

Em um estudo com idosos com sequela de AVC, Lee J e Seo K (2014), relata que a realização de treinamento do padrão de membro inferior usando o método FNP por quatro semanas promove uma melhora na capacidade de equilíbrio dinâmico e no treinamento de marcha em rampa, ao se comparar o grupo experimental e o controle através dos valores obtidos no teste de alcance funcional, contribuindo com os autores já citados.

No estudo idealizado por Sharma V e Kaur J (2017), havia a participação de vinte e três pacientes acometidos pelo AVC, foram recrutados e alocados aleatoriamente em dois grupos, sendo que o primeiro utilizou fortalecimento combinado de tronco com FNP e o segundo grupo utilizou FNP pélvico com exercícios de flexibilidade do tronco.

O desempenho de ambos os grupos foi avaliado na Escala de Comprometimento de Tronco (ECT), Avaliação de Mobilidade Orientada ao Desempenho de Tinetti (Tinetti-POMA), Teste de Sistemas de Avaliação de Equilíbrio (Mini-BESTest), Escala de Marcha de Wisconsin (EMW) e Índice de Atividades da Vida Diária de Barthelantes e após a conclusão da intervenção.

Sobre o estudo de Sharma $\mathrm{V}$ e Kaur $\mathrm{J}$ (2017), houve uma melhora significativa nos escores da TinettiPOMA, Mini-BES Test e Barthel no grupo do FNP pélvico mais flexibilidade de tronco que pode ser justificada pelas técnicas de FNP pélvico que atuam facilitando o mecanismo neuromuscular dos movimentos da pelve durante a marcha. Através da aplicação de alongamento, uso de padrões de movimento específicos, resistência máxima, proporcionaria resultados como a melhora do controle de tronco, e equilíbrio durante a marcha.

Kumar S, et al. (2012), avaliaram também os efeitos da técnica do FNP pélvico através dos parâmetros da marcha e na mobilidade funcional no hemiparético, com protocolo de atividades por 30 minutos diários, 3 dias por semana durante 4 semanas e constataram que o FNP pélvico tem efeito significativo na funcionalidade a marcha do paciente.

No estudo de Kyochul SEO, et al. (2015), foram avaliados pacientes acometidos pelo AVC onde o objetivo foi analisar a capacidade de desempenho do equilíbrio por meio do treinamento da marcha, as avaliações se deram através da EBB (Escala de equilíbrio de Berg), do TUG (Time up and go) e do teste de alcance funcional.

Os resultados no grupo experimental mostraram um aumento significativo no EEB e FRT e uma redução no tempo de execução do TUG, já no grupo controle não houve mudanças estatísticas nos testes. Utilizando as mesmas escalas de avaliação e metodologias semelhantes, Dietz B (2006), mostra em seu estudo que os valores de EBB, FRT e TUG do grupo controle não apresentaram significância, além de não haver diferença significativa entre os dois grupos após a intervenção. $O$ autor justifica isso pelo treino de marcha em sua pesquisa ser em rampas.

\section{CONSIDERAÇÕES FINAIS}

O estudo teve como objetivo, analisar as evidências cientificas encontradas na literatura, sobre o tratamento da eletroestimulação funcional (FES) e da facilitação neuromuscular proprioceptiva (FNP) em pacientes com sequelas de AVC, alcançou seu propósito, já que os estudos mostraram evidências plausíveis acerca da superioridade dos grupos experimentais sobre os grupos controle. Com a aplicação dos dois recursos, foi observado que os mesmos promoveram uma reabilitação mais eficaz, proporcionando maior qualidade de vida aos pacientes. Sugere-se que estudos mais complexos sejam realizados, com amostras maiores e mais homogêneas, na tentativa de análises mais eficientes. 


\section{REFERÊNCIAS}

1. ALON G, et al. Functional Electrical Stimulation Enhancement of Upper Extremity Functional Recovery During Stroke Rehabilitation: A Pilot Study. Neurorehabilitation and Neural Repair. 2007; 21(3).

2. BARBOSA DJ, et al. Recuperação após acidente vascular cerebral em adulto jovem submetido à fisioterapia alternativa. Revista Interfaces: Saúde, Humanas e Tecnologia. 2015; 2(6).

3. BOHANNON RW. Motricity index scores are valid indicators ofparetic upper extremity strength following stroke. Journal of Physical Therapy Science. 1999; 11: 59-61.

4. BRASHEAR A, et al. Inter- and intra rater reliability of the Ashworth scale and disability assessment scale in patients with upper-limbpos tstroke spasticity. The Archives of Physical Medicine and Rehabilitation. 2002; 83: 1349-54.

5. BRASIL. Ministério da saúde. Acidente vascular cerebral, 2019. Disponível em: http://portalms.saude.gov.br/saudede-a-z/acidente-vascular-cerebral-avc. Acesso em: 20 mar. 2019.

6. BRASIL. Ministério da saúde. Acidente vascular cerebral. 2017. Disponível em: http://portalms.saude.gov.br/saudede-a-z/acidente-vascular-cerebral-avc. Acesso em: 14 abr. 2019.

7. CHAN ML, et al. Bilateral Upper Limb Training With Functional Electric Stimulation in Patients With Chronic Stroke. Neurorehabilitation and Neural Repair. 2009; 23(4): 357-365.

8. DIETZ B. International PNF Basic Course Book. Gwangjoo; 2006.

9. GARLAND SG, et al. Postural responses to unilateral arm perturbation em Young, elderly, and hemiplegic subjects. The Archives of Physical Medicine and Rehabilitation. 1997; 78: 1072-1077.

10. GIL AC. Como elaborar projetos de pesquisa. São Paulo: Atlas; 2010.

11. KARAKUS D, et al. Effects of Functional Electrical Stimulation on Wrist Function and Spasticity in Stroke: A Randomized Controlled Study. Turkish Journal of Physical Medicine and Rehabilitation. 2013; 59: 97-102.

12. KIM Y, et al. The effects of trunk stability exercises using PNF on the functional reach test and muscle activities of stroke patients. Journal of Physical Therapy Science. 2011; 23(5): 699-702.

13. KUMAR S, et al. Effect of PNF technique on gait parameters and functional mobility in hemiparetic patients. Journal of Exercise Science and Physiotherapy. 2012; 8: 67-73.

14. KYO CSC, KIM H. The effects of ramp gait exercise with PNF on stroke patients' dynamic balance. Journal of Physical Therapy Science. 2015; 27: 1747-1749.

15. KYOCHUL SEO, et al. The effects of stair gait training using proprioceptive neuromuscular facilitation on stroke patients' dynamic balance ability. Journal of Physical Therapy Science. 2015; 27: 1459-1462.

16. LARCEDA NN, et al. Efeitos da facilitação neuromuscular proprioceptiva na estabilidade postural risco de quedas em pacientes com sequela de acidente vascular encefálico: estudo piloto. Fisioterapia e Pesquisa. 2013; 20(1): 37-42.

17. LEE J, SEO K: The effects of stair walking training on the balance ability of chronic stroke patients. Journal of Physical Therapy Science. 2014; 26: 517-520.

18. MAGDALON EC. Facilitação neuromuscular proprioceptiva: tratamento isolado em comparação com a associação da estimulação elétrica neuromuscular em membro superior de pacientes hemiparéticos pós-AVC. Dissertação (Mestrado) - Universidade Estadual de Campinas, São Paulo, 2004.

19. MANGOLD S, et al. Motor Training of Upper Extremity With Functional Electrical Stimulation in Early Stroke Rehabilitation. Neurorehabilitation and Neural Repair. 2009; 23(2): 184-190.

20. MARTIN ST, KESSLER M. Neurologic interventions for physical therapy. 2. ed. Missoure: Saunders; 2007.

21. MILESKI ME, et al. Efeitos da eletroestimulação e da facilitação neuromuscular proprioceptiva na marcha de hemiparéticos. Revista Ciência e Saúde. 2013; 6(1): 29-36.

22. PIOVESAN A, TEMPORINI ER. Pesquisa exploratória: procedimento metodológico padrão estudo de fatores humanos no campo da saúde pública. Revista de Saúde Pública. 1995; 29(4).

23. RATNASABAPATHY Y, et al. Shoulder pain in people with a stroke: a population-based study. Clinical Rehabilitation. 2003; 17(3): 304-311.

24. SANTOS NS, et al. Facilitação neuromuscular proprioceptiva na marcha em pacientes com sequela de acidente vascular encefálico. Arquivo de Ciências e Saúde. 2016; 23(2): 87-91.

25. SHARMA V, KAUR J. Effect of core strengthening with pelvic proprioceptive neuromuscular facilitation on trunk, balance, gait, and function in chronic stroke. Journal of Exercise Rehabilitation. 2017; 13(2): 200-205.

26. SHEARD PW, PAINE TJ. Optimal contraction intensity during proprioceptive neuromuscular facilitation for maximal increase of range of motion. The Journal of Strength and Conditioning Research. 2010; 24(2): 416-21.

27. SHEPHERD RB. Exercise and training to optimize functional motor performance in stroke: driving neural reorganization?. Neural plasticity. 2001; 8.

28. SOUZA MT, et al. Revisão integrativa: o que é e como fazer. Einstein. 2010; 8(1): 102-106.

29. TAUB $E$, et al. Technique to improve chronic motor deficit after stroke. The Archives of Physical Medicine and Rehabilitation. 1993; 74: 347-354. 